\section{Aprender estratégicamente: Percepciones de docentes y alumnos de un programa de maestría a distancia}

\author{
Elizabeth Salcedo Lobatón \\ Pontificia Universidad Católica del Perú (Perú)
}

\section{Resumen}

En pleno siglo $X X I$, en un contexto de cambio tecnológico en que se produce diariamente información y conocimiento nuevo, una competencia transversal fundamental por fortalecer en la formación universitaria, principalmente a distancia, es el aprendizaje estratégico $(A E)$ de los estudiantes. Se propone una noción operacional de $A E$, que integra en una sola categoría las características del aprender a aprender, del aprendizaje autónomo y autorregulado. Aunque estas categorías observan fundamento y consenso académico, no necesariamente están integradas a los perfiles de competencias que orientan los programas de formación.
Learning strategically: Perceptions of teachers and students of a distance learning master program

\section{Elizabeth Salcedo Lobatón}

Pontificia Universidad Católica del Perú (Perú)

\begin{abstract}
In the 21st century, in a context of technological change in which increasing amounts of new information and knowledge is produced on a daily basis, it is considered that a fundamental transversal skill to be strengthened in universities, especially in distance education, is students' strategic learning. The research proposes an operational notion of strategic learning, which integrates in a single category, the characteristics of learning to learn, lifelong learning and self-regulated learning. Although these categories hold basis and consensus in the academic community, they are not necessarily integrated into the competency profiles that guide the educational programs.
\end{abstract}


En una maestría a distancia, que forma profesionales tomadores de decisiones en políticas públicas, se realizó una consulta sobre la necesidad de fortalecer capacidades para aprender estratégicamente. Docentes, alumnos y diseñadores instruccionales expresaron su opinión sobre la relevancia de esta competencia, sobre las capacidades fundamentales que deberían priorizarse para mejorar el perfil de los egresados, sobre el nivel de avance de estas capacidades en alumnos que estudian la maestría, y sobre la responsabilidad que tiene un programa de formación de posgrado en dicha materia. El estudio de caso aporta una visión crítica sobre la incorporación del AE como capacidad transversal en programas de educación superior y de posgrado a distancia, como alternativa para enfrentar los déficits que provienen de la educación básica, del propio perfil de los alumnos y de las debilidades de los procesos de enseñanza a distancia en el nivel universitario.

Palabras clave: Aprendizaje estratégico, educación a distancia, aprendizaje autónomo, aprendizaje autorregulado, educación superior, aprender a aprender.
A consultation about the need to strengthen the strategic learning skills has been conducted in a distance learning master program, responsible for the education of public policies decision-makers. Teachers, students and instructional designers have expressed their opinion about the relevance of this competency, about the fundamental skills that should be given priority to improve the profile of the graduate students, about the students' level of achievement in these skills and about the degree of responsibility the educational post graduate program has in this matter. The case study contributes with a critical view about the incorporation of strategic learning as a transversal skill, especially in distance learning programs, in order to face the deficits drawn from basic education, from the own profiles of students, and from the deficiencies of undergraduate teaching processes.

Key words: Strategic learning, distance learning, lifelong learning, self-regulated learning, higher education, learning to learn.

En el contexto de cambio tecnológico y el auge de los paradigmas del nuevo siglo: sociedad de la información, sociedad del conocimiento, y sociedad del aprendizaje, y de las exigencias que conllevan estas transformaciones, diversas reflexiones (Unesco, 1998; Pozo, Monereo y Castelló, 2001; Beneitone, Esquetini, González, Marty Maleta, Siufi y Wagenaar, 2007; González y Cabrera, 2013; Monereo y Badia, 2013; Pozuelo, 2014; etc.) han expresado la necesidad de cambios y adecuaciones en el sistema educativo, en los procesos de enseñar y aprender, sugiriendo el desarrollo de nuevas competencias para aprender a aprender a lo largo de la vida.

Esta especial atención hacia el manejo de estrategias de aprendizaje autónomo como planificar, autorregular y autoevaluar el propio proceso de aprender, ha merecido una creciente revisión de cara a una sociedad en la que el conocimiento se ha convertido en una mercancía preciada. 
Sin embargo, estas reflexiones han estado predominantemente asociadas a la educación básica y a la formación presencial, y no han cobrado tanta relevancia en el análisis de la educación superior a distancia.

En el Perú donde se ubica el estudio, la debilidad del sistema educativo y la comprobación empírica de que los alumnos que cursan educación superior y de posgrado no han alcanzado destrezas esenciales para un adecuado tránsito por el proceso académico, plantea la necesidad de revisar los procesos de aprendizaje que se producen en este nivel formativo.

Desde las características de la población universitaria y de posgrado se advierte también que la motivación por aprender no siempre está presente, y que es la presión del mercado laboral la que induce, mayormente a los jóvenes y adultos, a participar de los programas de formación. También se identifican debilidades en los procesos de selección y admisión de los estudiantes en el nivel de posgrado, lo cual conlleva a la matrícula de alumnos que no necesariamente poseen las capacidades mínimas para lograr un desempeño promedio. El problema cobra mayor relevancia en los procesos de formación a distancia, que aunque representan una oportunidad para democratizar la educación y facilitar el acceso a poblaciones más amplias, no siempre han merecido una renovación constante de la forma de enseñar y de aprender para garantizar eficacia educativa.

La nueva Ley Universitaria Peruana, en proceso de implementación, no ha validado la educación virtual como una modalidad de calidad aceptable, equivalente a la formación presencial para la formación de posgrado. Este hecho reta doblemente a la educación a distancia a explorar su desempeño y a plantearse nuevas exigencias para orientar los procesos de aprendizaje.

El aprender, visto de manera general, sugiere un proceso cognitivo similar en la dimensión presencial y a distancia. No obstante, la constatación empírica nos acerca a algunas particularidades inherentes al proceso de aprender a distancia que merecen especial atención, porque exigen algunas capacidades específicas de alumnos y docentes que no siempre son contempladas desde los programas formativos. El estudio de caso se ha preguntado:

¿Qué capacidades transversales se deben potenciar en los alumnos del Programa de Maestría en Gerencia Social a Distancia (MGS-D), para que fortalezcan su forma de aprender $(A E)$, y puedan enfrentar con éxito las demandas de conocimiento que les exige el contexto?

El estudio se propuso:

- Identificar las capacidades específicas de AE que son consideradas fundamentales para la formación y desempeño de los alumnos de la MGS-D (validación de un perfil).

- Conocer la autopercepción de los estudiantes sobre el nivel de desarrollo de estas capacidades.

- Conocer la percepción de docentes y estudiantes sobre la responsabilidad que tiene la universidad y ellos mismos en el desarrollo de estas capacidades. 


\section{Paradigmas que aportan a la noción de aprender estratégicamente}

Diversas teorías psicológicas y pedagógicas han contribuido a la noción de AE. Uno de los paradigmas centrales ha sido el aprender a aprender, incorporado por la Comisión Europea como una de las ocho competencias básicas del sistema europeo, definida como "La capacidad para proseguir y persistir en el aprendizaje, organizar el propio aprendizaje, realizar un control eficaz del tiempo y la información, individual y grupalmente" (Martín, 2008:72).

Aprender a aprender no se refiere al aprendizaje directo de contenidos sino al aprendizaje de habilidades para asimilar contenidos (Beltrán, 1993, citado por Monereo, Castelló, Clariana, Palma y Pérez, 1999), significa a la vez capacidad y compromiso de las personas para construir su propio saber a partir de su experiencia de vida y de las oportunidades de aprender, bajo la finalidad de utilizar y aplicar el conocimiento en los diversos contextos donde se desarrolla. Competencia para algunos, estrategia para otros, implica desarrollar aspectos cognitivos y emocionales, además de determinadas capacidades metacognitivas (Martín, 2010), que permiten a la persona lograr conciencia de cómo aprender (Fernández y Wompner, 2007), además conocer y regular sus propios procesos de aprendizaje.

La competencia implica el desarrollo central de capacidades procedimentales como obtener, procesar y asimilar nuevos conocimientos, así como algunas habilidades de búsqueda de información y la utilización de guías de estudio o manuales; la internalización de reglas y procedimientos que deberán ser explícitas para autorregular su proceso y el desarrollo de la autoestima para que el estudiante acepte el rechazo que puede provocar el error, y el manejo de la tensión que implica el esfuerzo de aprender.

Otro paradigma es el aprendizaje autónomo, concepto de alta relevancia para la educación presencial y también virtual. En esta última perspectiva de educación mediadas por las TIC, tres autores de obligada referencia: Delling (1966), Wedemeyer $(1972,1979)$ y Moore (1983), citados por Stojanovic (1994), la han referido como una cualidad casi inherente señalando que el estudio autónomo permite a las personas acceder a un aprendizaje con ritmo propio, en los tiempos que disponen, desde el lugar donde se encuentren. Sin embargo, no todos los programas virtuales ofrecen las mismas condiciones (enseñanza, recursos tecnológicos, etc.), de allí que las estrategias didácticas y actividades formativas para aprender a distancia cobren extrema importancia para alcanzar o no el aprendizaje autónomo, sin olvidar que las características del propio estudiante son un factor más a tener en cuenta.

Desde una concepción más amplia, vinculada a la educación presencial, Argüelles y Nagles (2009) consideran que el aprendizaje autónomo es un proceso que estimula al estudiante para que sea el autor de su propio desarrollo, lo motiva a construir por sí mismo el proceso a seguir para lograr el conocimiento que ignora. Estos mismos autores precisan que la competencia de estudio autónomo integra varias áreas de capacidad: cognitiva (manejo de procesos y estrategias), socioafectiva (expresión de afectos, actitudes, valores) y motora (expresión de la habilidad) y aunque no hacen alusión a las capacidades metacognitivas que sí considera el "aprender a aprender", es tácito que se requiere de ellas para posibilitar este comportamiento. 
Aebli (1991), Argüelles y Nagles (2009), Santos y Cámara (2010), González (2011) y Bacchetta (2013) han formulado importantes aportes que no solo caracterizan y fundamentan la autonomía como una cualidad deseable para la educación actual, sino que describen cómo opera el proceso en las personas para el logro de esta capacidad, las condiciones que deben darse para que ello suceda y el tipo de actividades y estrategias que favorecen su logro.

Para asumir un aprendizaje autónomo, un alumno debe: a) Establecer contacto, por sí mismo, con cosas e ideas; b) Comprender por sí mismo fenómenos y textos; c) Planear por sí mismo acciones y solucionar problemas; d) Ejercitar actividades por sí mismo, manejar información; e)mantener por sí mismo la motivación para la actividad y el aprendizaje.

El aprendizaje autónomo supone una capacidad de "filtrar conocimiento socialmente construido" (MacDougall, 2010:50), lo cual implica un sistema conceptual muy personal, que más adelante le sirva al estudiante para seleccionar información útil a sus objetivos, lo que ha sido llamado por varios autores como personalización del aprendizaje.

Existe un compromiso efectivo del estudiante en el proceso de aprendizaje. Esta característica, aludida por diversos autores, hace la diferencia en la calidad de su participación, que se ve paradójicamente afectada en tiempos en donde jóvenes y adultos acceden a diversos medios tecnológicos con fines de ocio, pero si se trata de obtener información y conocimiento, surge una sensación de aislamiento y abandono (distancia transaccional) (Santos y Camara 2010), que sin autonomía impide concretar el objetivo. Aquí aparece la necesidad de la capacidad metacognitiva para hacer conciencia sobre cómo aprender, para decidir (en soledad) las formas más efectivas de participación y cumplir con la aplicación de los procedimientos de aprendizaje.

Otro paradigma es la autorregulación: forma de educación sustentada en la voluntad y en la destreza de los estudiantes (Blumenfeld y Marx, 1997; McCombs y Marzano, 1990; citados por Torrano y Gonzales, 2004). Es una educación que ayuda a hacer consciencia de cómo opera el pensamiento, y a ser estratégicos para el logro de metas de saber. Es un aprendizaje activo y constructivo por el cual el estudiante establece sus propios objetivos de aprendizaje, procurando monitorizar, regular y controlar sus pensamientos (Zimmerman, 2002).

A partir de Torrano y Gonzales (2004), Zulma (2006), y Cerezo, Núñez, Fernández, Suárez, y Tuero (2011), se han recogido tres tipos de planteamientos sobre autorregulación del aprendizaje: los que asumen que el concepto de autorregulación y metacognición se refieren casi a los mismos procesos; los que asumen que la autorregulación es un componente de la metacognición; y aquellos planteamientos que integran ambos conceptos bajo lo que se ha denominado aprendizaje regulado.

Los estudiantes que autorregulan su aprendizaje:

- Conocen y saben emplear estrategias cognitivas (elaboración, organización) que les ayudan a transformar, organizar, elaborar y recuperar información. 
- Saben planificar controlar y dirigir sus procesos mentales hacia el logro de metas personales (metacognición).

- Observan motivación y emoción asociadas a la autoeficacia en las tareas académicas (disfrutan el logro de tareas).

- Planifican y controlan el tiempo y el esfuerzo que emplean en la tarea.

- Ponen en marcha estrategias volitivas para evitar distracciones externas y mantener la concentración.

El modelo, Pintrich (2000) citado por Zulma (2006), y Gonzales (2004), integra los diversos procesos y actividades que favorecen la autorregulación en cuatro áreas: la cognición, la motivación, el comportamiento y el contexto, aunque no queda muy claro cómo el estudiante alcanza a regular estos últimos factores que no controla.

La gestión autónoma y autorregulada implica también competencias de gestión, planificación competencias cognitivas superiores, y competencias de gestión de la calidad y la innovación (Martín Cuadrado, 2011).

Aunque se proponen discusiones teóricas y controversias que hacen inacabado el concepto, desde un sentido práctico, la autorregulación del aprendizaje parece central en la conducción y control del proceso de aprender, cualidad indispensable en los estudios a nivel superior y a distancia. Desde esta última perspectiva Monereo y Badia (2013) señalan que las tecnologías y medios de enseñanza virtual pueden hacer posibles nuevos procesos de autorregulación y corregulación del aprendizaje, a través de indicaciones reflexivas: el feedback de una tarea o la interacción con un "meta-tutor" en plataformas programadas. En los tres casos observamos el importante rol del proceso de enseñanza y el diseño instruccional. Los autores también expresan la importancia de las tareas de aprendizaje colaborativo, como parte de un sistema de autorregulación grupal para la planificación, supervisión y evaluación de procesos de aprendizaje.

Otro paradigma aportado por Monereo (1999) es la noción de "estrategia de aprendizaje", concebida en los años ochenta, como parte de las teorías cognitivas y definidas como un conjunto de procesos mentales, operaciones y procedimientos que facilitan la codificación, adquisición, retención y evocación de información. Luego con la influencia de las teorías psicológicas socioculturales, la estrategia de aprendizaje integró los aspectos sociales y culturales vigentes hasta hoy. Como parte de este desarrollo surge el $A E$ que introduce la idea de intencionalidad que guía las acciones de aprendizaje hacia las metas de conocimiento. Para ello el AE debe ser planeado en relación con las condiciones del contexto (Pozo, Monereo y Castelló, 2001).

El AE no está referido solo al uso de estrategias de aprendizaje: activación intencional y deliberada de unos conocimientos conceptuales, procedimentales o actitudinales (Monereo y Badia, 2013), concepto más difundido (Monereo, 2001; Díaz, 2002; citados por Huertas, 2007) sino que involucra procesos cognitivos y motivacionales que promueven el aprendizaje significativo de los alumnos, es decir, el aprendizaje con sentido. Por ello, para Gargallo (2011) aprender estratégicamente supone indistintamente funcionamiento autónomo y autor-regulado, noción compartida desde esta propuesta. La diferencia fundamental entre el AE y la sola aplicación de estrategias de aprendizaje, 
parece estar en la idea del llamado "control estratégico" (Monereo y Badia, 2013), que aporta un valor agregado al uso de conocimientos conceptuales y técnicos para aprender.

Una secuencia adecuada para la instrucción estratégica, implica una transferencia progresiva del control de la tarea, del profesor al alumno, de forma que este tenga cada vez más autonomía y responsabilidad en su aprendizaje (Monereo y Badia, 2013).

Desde investigaciones referidas principalmente al sistema escolar Badia y Monereo (2008) han señalado que el $A E$ no ha logrado convertirse aún, en la columna vertebral de las programaciones educativas. En el medio universitario y en la modalidad virtual, hay poca comprobación empírica sobre el tema. Desde las preocupaciones actuales de la educación superior, y en la modalidad a distancia, interesa a este estudio plantear el AE como una noción integradora de varios paradigmas contemporáneos, capaz de orientar procesos de enseñanza aprendizaje a distancia.

\section{Aprender estratégicamente: capacidad fundamental en educación superior a distancia}

Desde el análisis de las capacidades genéricas deseables en la educación superior, hay un debate abierto sobre cuáles son aquellas dimensiones claves que deben ser incorporadas en la currícula universitaria. Desde espacios europeos y latinoamericanos se han desarrollado reflexiones y propuestas de reforma educativa que han impactado positivamente en algunas universidades, promoviendo el debate e incorporación de capacidades transversales, entre ellas algunas vinculadas al aprender estratégicamente (motivación para aprender, capacidades metacognitivas, planificación y autorregulación, entre otras). No obstante, existe poca aplicación e investigación empírica sobre la transversalización de tales capacidades en programas de educación superior y de posgrado.

En programas de educación a distancia con frecuencia se asume que el manejo de un estudio más autónomo, autorregulado y por tanto estratégico es casi una característica propia que traen los alumnos a este nivel de formación, o bien se estima que son características favorecidas por la educación mediada por las TIC sin contemplar la relevancia que tiene el diseño instruccional, el rol docente, y las propias características de cómo aprende el estudiante.

Numerosos estudios citados Meng-Jung Tsai (2009) observaron que la estrategia de aprendizaje (principalmente metacognitiva) es uno de los factores que más influye en el proceso. Se ha referido que la búsqueda de información, selección, sistematización, en línea, son procesos complejos y difíciles para los estudiantes.

En el caso que se presenta, el $A E$ se asume como una noción articuladora de diversas áreas de capacidad. Es una acción activa del estudiante en el proceso de enseñanza-aprendizaje en un contexto determinado.

El AE alude a una acción activa del estudiante en el proceso de enseñanza aprendizaje, en un contexto determinado. No se refiere solo al uso de estrategias para aprender, sino que supone visionar, pensar y actuar estratégicamente, teniendo cuyo fin es lograr determinados saberes y bajo una motivación superior. Ello implica tener 
conciencia clara de la necesidad de aprender, conocimiento de los diversos factores que intervienen en la construcción de los saberes, intencionalidad y decisión propia.

Aunque la conducta del estudiante es central en este concepto porque es allí donde se medirán los cambios y resultados, aprender estratégicamente supone no solo un aprendiz con habilidades diferentes (autonomía, autorregulación, motivación, entre otros), sino una "doctrina" (Sun, 2009) clara, un proceso de enseñanza direccionado en el mismo sentido, y docentes con pleno conocimiento del proceso de aprendizaje y de cómo influirlo significativamente. La enseñanza estratégica también es proceso intencional.

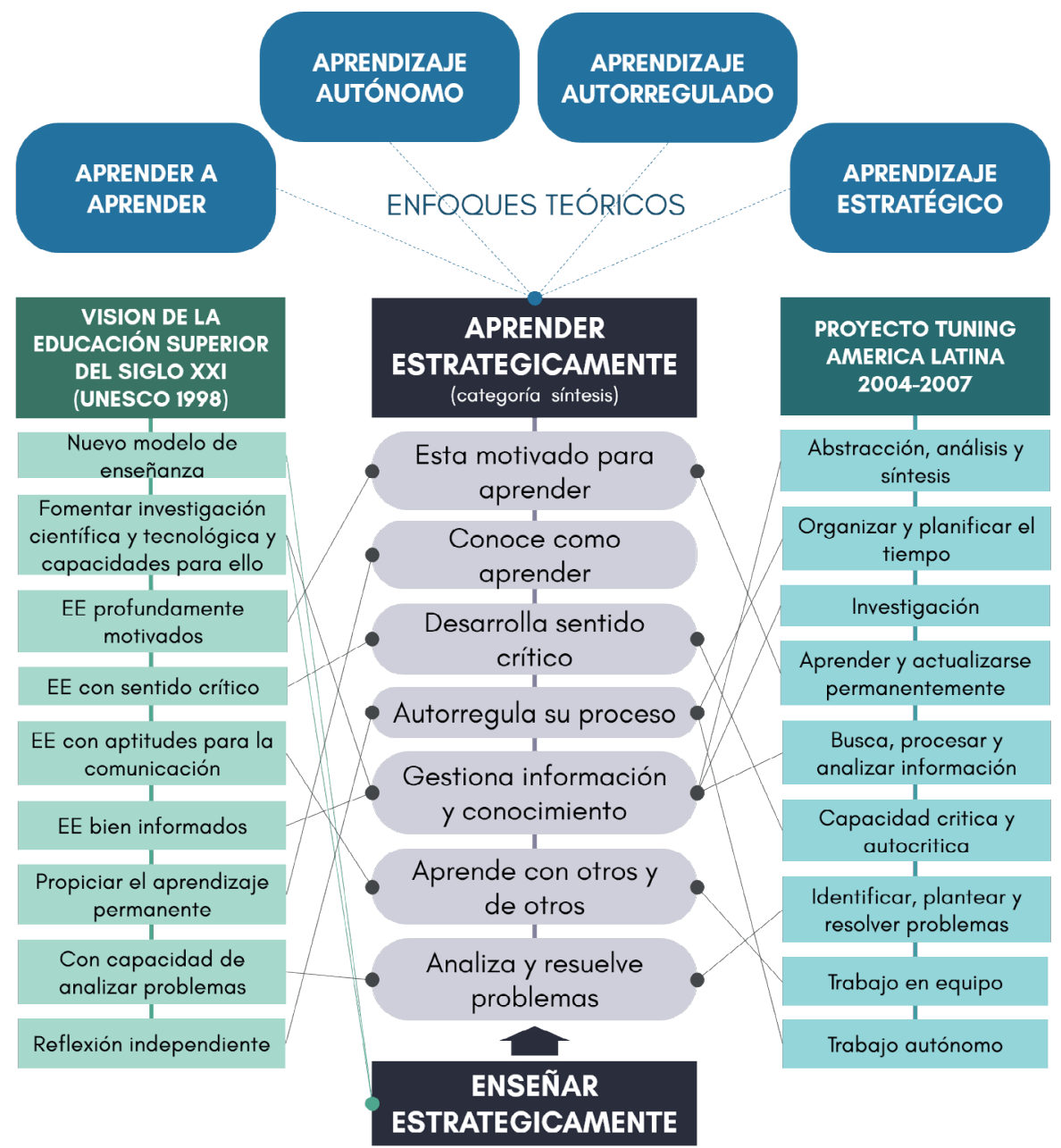

Figura 1. AE: Propuesta de capacidades transversales orientadoras para la formación universitaria. Fuente: Elaboración propia.

El AE se ubica en una perspectiva constructivista y sociocultural, que orienta a un aprendizaje más efectivo, rescata una visión sistémica del proceso de aprender, involucra factores inherentes al estudiante y a otros agentes educativos que interactúan con él y con su entorno e incorpora habilidades de reflexión, de planificación, técnicas de búsqueda de información, habilidades de autoevaluación, entre otras sugeridas por MacGregor (1999) citado por Meng-Jung Tsai (2009).

La propuesta operacional de AE (Figura 1), puesta a consulta en el programa de formación de la MGS-D parte de las necesidades y retos de la educación superior 
propuestos por Unesco desde fines del siglo pasado (Unesco, 1998); también integra nueve de las veinticinco capacidades genéricas propuestas por el Proyecto Tuning para América Latina, y propone siete áreas de capacidad que se operacionalizan en diecisiete dimensiones específicas.

\section{Metodología}

Es una investigación aplicada, orientada a aportar a la mejora continua del programa y ha optado por la modalidad de estudio de caso intrínseco (Stake, 1998), caracterizado por el examen sistemático y a profundidad del problema de estudio.

Aunque el objeto de conocimiento es esencialmente cualitativo, el estudio mereció un tratamiento metodológico mixto que combino la aplicación de un cuestionario semiestructurado que permitió la consulta a alumnos y docentes sobre la valoración de dimensiones organizadas en escalas cerradas, combinando a su vez la aplicación de entrevistas a profundidad con docentes, que permitió el recojo de relatos, descripciones y discursos.

El artículo resume dos de las tres fases del proceso de conocimiento realizado:

Fase 1: Revisión teórica y recuperación de algunos estudios empíricos relacionados al aprendizaje estratégico, en la idea de identificar y construir un menú de capacidades genéricas fundamentales que permitieron la construcción de una noción operacional del $A E$, a ser utilizada en la investigación. El producto de esta primera fase fue la construcción de una propuesta de capacidades genéricas (perfil de capacidades).

Fase 2: Validación de una propuesta de perfil de capacidades para el $A E$, que distingue un momento de validación técnica, y el proceso de consulta a la comunidad educativa involucrada en el programa. De esta fase se ha obtenido como producto un perfil de capacidades genéricas deseables para orientar al programa académico hacia un AE. Esta etapa ha permitido recoger comparativamente la valoración que asignan principalmente alumnos, docentes a las diversas capacidades que deben potenciarse en la formación profesional del programa de estudios, dando cuenta de visiones, paradigmas, valores y prioridades de la formación profesional a distancia, que no siempre son recogidas por el diseño de los programas.

Fase 3: Validado el perfil, el proceso de investigación avanzó en el análisis de diversos aspectos del proceso de enseñanza y el aprendizaje virtual de los alumnos del programa académico y su relación y aporte al perfil de $A E$ validado.

La maestría, elegida como unidad de análisis, es el programa a distancia de mayor antigüedad en la PUCP. Es un programa interdisciplinario, dirigido a formar profesionales tomadores de decisiones en políticas públicas.

En la tabla siguiente (Tabla 1) se precisa los informantes que participaron del proceso de consulta y las técnicas utilizadas. 
Tabla 1. Informantes y técnicas utilizadas.

\begin{tabular}{|l|l|l|l|l|}
\hline $\begin{array}{l}\text { Tipo de } \\
\text { informante }\end{array}$ & $\begin{array}{l}\text { Universo de } \\
\text { estudio }\end{array}$ & Criterios de elección & Participantes & Técnica aplicada \\
\hline Estudiantes & 157 & $\begin{array}{l}\text { Alumnos matriculados en cuatro o } \\
\text { más ciclos académicos. }\end{array}$ & $\begin{array}{l}\text { Cuestionario } \\
\text { online }\end{array}$ \\
\hline $\begin{array}{l}\text { Profesores } \\
\text { coordinadores }\end{array}$ & 26 & $\begin{array}{l}\text { Profesores de cursos obligatorios, } \\
\text { que participan del diseño } \\
\text { instruccional. }\end{array}$ & 14 & $\begin{array}{l}\text { Entrevista a } \\
\text { profundidad }\end{array}$ \\
\hline $\begin{array}{l}\text { Gestores y } \\
\text { asesores }\end{array}$ & 4 & Gestores y asesores académicos. & 4 & $\begin{array}{l}\text { Entrevista a } \\
\text { profundidad }\end{array}$ \\
\hline
\end{tabular}

La población estudiantil está compuesta por profesionales predominantemente mujeres (59\%), entre treinta y cuarenta años (54\%) que provienen de todo el país. El $34 \%$ procede de Lima (34\%), mientras que el $66 \%$ lo hace desde las regiones. El $65 \%$ realizó estudios de pregrado en universidades públicas y casi la mitad se han formado en carreras de ciencias humanas (24\%) y ciencias sociales (24\%). Alrededor del $20 \%$ ocupa cargos gerenciales. Su principal motivación para estudiar la maestría se relaciona con la necesidad de fortalecer su desempeño en el campo social. La mayor parte de alumnos eligieron estudiar a distancia debido a la distancia geográfica de la PUCP.

De los docentes de la maestría, el 77\% tiene el grado de Magister y el $26 \%$ el grado de Doctor. El $66 \%$ han desarrollado sus estudios de posgrado en la MGS-PUCP. EI $82 \%$ de los docentes obtuvo su grado o título de posgrado en la misma universidad y solo el $18 \%$ se graduó en universidades extranjeras.

\section{Resultados}

Docentes y alumnos consultados valoraron positivamente la propuesta de integrar a la currícula de estudios la mayor parte de las capacidades de AE propuestas.

Acá (en la maestría virtual) se desarrolla mucho la responsabilidad del alumno por aprender (...) El alumno tiene que acercarse y tratar de captar (...) una educación estratégica, obliga al alumno a participar más activamente de su educación, fuerza a la persona a actuar sobre su educación (DC8).

Para mí hay grandes vacíos en la gente que viene acá, por supuesto no es culpa de la universidad (...) Muchos tienen una cultura general muy poco deseable... Ni la escuela, ni la propia universidad de pregrado, se preocupan por proveer al alumno de una base cultural mínima (DC9).

Si incorporamos $\mathrm{AE}$, la maestría puede ayudar a poner el piso más plano, y a tener habilidades para hacer docencia a distancia ya que hay algunos docentes no hemos hecho cursos para enseñar a distancia (...) Se trata de que todos tengamos habilidades desarrolladas para que podamos hacer un mejor trabajo (DC12).

Debe darse un aprendizaje un poco más contextualizado, y más autorregulado (...) Que sea el propio chico el que estudie. Pero nadie se atreve a mover el elefante que está instalado ahí (DC6). 


\section{Capacidades de AE fundamentales en el proceso de formación}

Se han consolidado las valoraciones de alumnos y docentes respecto de las diecisiete capacidades consultadas. Este insumo aporta a la construcción de una propuesta instrumental (perfil) pero principalmente a explorar concepciones presentes en alumnos y docentes que requieren ser trabajadas en el marco de la optimización de los procesos de enseñanza y aprendizaje.

Tabla 2. Valoraciones de docentes y alumnos sobre la importancia de las capacidades AE en la formación de los alumnos de la MGS-D.

\begin{tabular}{|c|c|c|c|c|c|}
\hline $\begin{array}{l}\text { Áreas de } \\
\text { capacidad }\end{array}$ & $\begin{array}{l}\text { Capacidades del alumno que } \\
\text { aprende }\end{array}$ & \multicolumn{2}{|c|}{ Docentes } & \multicolumn{2}{|c|}{ Alumnos } \\
\hline \multirow[t]{3}{*}{$\begin{array}{l}\text { Motivación } \\
\text { para } \\
\text { aprender }\end{array}$} & $\begin{array}{l}\text { Es consciente de la alta demanda } \\
\text { de conocimiento que deriva del } \\
\text { actual contexto }\end{array}$ & $100 \%$ & & $89 \%$ & $11 \%$ \\
\hline & $\begin{array}{l}\text { Tiene claro los objetivos y metas } \\
\text { de conocimiento que desea } \\
\text { lograr. }\end{array}$ & $100 \%$ & & $86 \%$ & $14 \%$ \\
\hline & $\begin{array}{l}\text { Está interesado en obtener } \\
\text { siempre nuevo conocimiento. }\end{array}$ & $91 \%$ & & $76 \%$ & $24 \%$ \\
\hline \multirow[t]{2}{*}{$\begin{array}{l}\text { Conoce como } \\
\text { aprender }\end{array}$} & $\begin{array}{l}\text { Conoce su propia forma de } \\
\text { aprender y las estrategias más } \\
\text { eficaces para hacerlo. }\end{array}$ & $92 \%$ & $8 \%$ & $64 \%$ & $34 \% 2 \%$ \\
\hline & $\begin{array}{l}\text { Usa intencionalmente estrategias } \\
\text { propias para lograr conocimiento. }\end{array}$ & $100 \%$ & & $76 \%$ & $22 \% 2 \%$ \\
\hline \multirow[t]{3}{*}{$\begin{array}{l}\text { Autorregula } \\
\text { su } \\
\text { aprendizaje }\end{array}$} & $\begin{array}{l}\text { Autoevalúa su proceso de } \\
\text { aprendizaje, e identifica barreras } \\
\text { que le impiden avanzar en el } \\
\text { conocimiento. }\end{array}$ & $92 \%$ & & $66 \%$ & $34 \%$ \\
\hline & $\begin{array}{l}\text { Planifica, su proceso de estudio, } \\
\text { con conocimiento de lo que } \\
\text { implica estudiar a distancia. }\end{array}$ & $92 \%$ & & $66 \%$ & $32 \% 2 \%$ \\
\hline & $\begin{array}{l}\text { Programa y dosifica las } \\
\text { actividades de estudio, en función } \\
\text { de cargas laborales y personales. }\end{array}$ & $75 \%$ & $25 \%$ & $73 \%$ & $25 \% 2 \%$ \\
\hline \multirow{3}{*}{$\begin{array}{l}\text { Gestiona } \\
\text { información } \\
\text { y conoci- } \\
\text { miento }\end{array}$} & $\begin{array}{l}\text { Busca, selecciona, clasifica, } \\
\text { sistematiza información. }\end{array}$ & $92 \%$ & & $72 \%$ & $28 \%$ \\
\hline & $\begin{array}{l}\text { Maneja metodologías y técnicas } \\
\text { de investigación aplicada. }\end{array}$ & $73 \%$ & $18 \% 9 \%$ & $89 \%$ & $9 \% 2 \%$ \\
\hline & $\begin{array}{l}\text { Usa las diversas fuentes de } \\
\text { información existentes, aplicando } \\
\text { criterios de rigor científico. }\end{array}$ & $91 \%$ & & $84 \%$ & $14 \% 2 \%$ \\
\hline
\end{tabular}


Tabla 2, continúa de la página anterior

\begin{tabular}{|c|c|c|c|c|c|}
\hline $\begin{array}{l}\text { Áreas de } \\
\text { capacidad }\end{array}$ & $\begin{array}{l}\text { Capacidades del alumno que } \\
\text { aprende }\end{array}$ & Docent & & \multicolumn{2}{|c|}{ Alumnos } \\
\hline \multirow[t]{3}{*}{$\begin{array}{l}\text { Aprende con } \\
\text { otros y de } \\
\text { otros }\end{array}$} & $\begin{array}{l}\text { Establece interacciones positivas } \\
\text { con sus docentes: consulta, } \\
\text { solicita información, debate ideas. }\end{array}$ & \multicolumn{2}{|l|}{$100 \%$} & $74 \%$ & $22 \% 4 \%$ \\
\hline & $\begin{array}{l}\text { Valora la interacción con otros } \\
\text { alumnos y el trabajo colaborativo. }\end{array}$ & $92 \%$ & & $55 \%$ & $41 \% \quad 4 \%$ \\
\hline & $\begin{array}{l}\text { Hacer uso de redes sociales } \\
\text { y aplicaciones virtuales para } \\
\text { acceder y socializar información. }\end{array}$ & $82 \%$ & $18 \%$ & $60 \%$ & $40 \%$ \\
\hline $\begin{array}{l}\text { Desarrolla } \\
\text { sentido } \\
\text { Critico }\end{array}$ & $\begin{array}{l}\text { Es crítico ante el conocimiento } \\
\text { absoluto y ante el propio proceso } \\
\text { de enseñanza. }\end{array}$ & $85 \%$ & $15 \%$ & $79 \%$ & $17 \% 4 \%$ \\
\hline \multirow[t]{2}{*}{$\begin{array}{l}\text { Analiza y } \\
\text { resuelve } \\
\text { problemas }\end{array}$} & $\begin{array}{l}\text { Observa, identifica y comprende } \\
\text { problemas, genera alternativas y } \\
\text { selecciona soluciones idóneas. }\end{array}$ & $92 \%$ & $8 \%$ & $57 \%$ & $43 \%$ \\
\hline & $\begin{array}{l}\text { Identifica factores externos que } \\
\text { influyen en su desempeño y toma } \\
\text { medidas para neutralizarlas. }\end{array}$ & $100 \%$ & & $80 \%$ & $18 \% 2 \%$ \\
\hline
\end{tabular}

Muy necesario; Medianamente necesario; No necesario

- Sobre la "motivación por aprender" se ha identificado un mayoritario consenso entre alumnos y docentes que la reconocen como un paso inicial, necesario para aprender, en un contexto donde "los alumnos de posgrado no quieren aprender sino solo tener el título" (D8). Se observa que casi la cuarta parte de alumnos (24\%) relativizan la capacidad "interés en lograr siempre nuevo conocimiento".

- Sobre el "Conoce cómo aprender", se exhibe un acuerdo mayoritario sobre la importancia de capacidades metacognitivas, que según los docentes "solo la traen una minoría de los alumnos..." (D9). Llama la atención un $34 \%$ de alumnos que no consideran muy importante el "conocimiento de su forma de aprender" y el "conocimiento de estrategias para aprender" e inclusive un alumno descarta esta capacidad. Pero, sí demandan acciones docentes que tienen directa incidencia en el desarrollo metacognitivo:

Luego de una evaluación en línea nos deberían enviar el resultado con las respuestas correctas, a fin de medir las respuestas correctas y las erradas (A17).

Los profesores te colocan una baja nota y no sustentan por qué (A12).

- En el eje de autorregulación, la planificación y la autoevaluación fueron bien valoradas por los docentes y la mayoría de alumnos.

Como en toda tu vida, tú tienes que autoevaluarte para poder mejorar (DC2). 
Me parece que es clave que el alumno sea crítico y autocritico, ya que tendría que saber sus puntos débiles para poder plantear estrategias de auto mejora (DC4).

Sin embargo, más de un tercio de estudiantes relativiza la importancia de las capacidades de autorregulación, y un alumno las desestima (2\%).

- En gestión de la información hay un acuerdo mayoritario en la importancia de las capacidades propuestas. Sin embargo, algunos docentes restan valor al manejo de metodologías y técnicas de investigación aplicada. Las razones que argumentan se sustentan en las diversas ideas que tienen del perfil del egresado, lo cual evidencia que no hay conocimiento claro del perfil, ni unidad de criterio sobre el mismo.

- En relación con la capacidad de aprender con otros observamos menor consenso. Los alumnos asignan una baja valoración al trabajo colaborativo (ver testimonio) y los docentes hacen lo propio con la capacidad de "usar redes sociales".

(...) Se me hace difícil encontrar un buen grupo ya que no conozco los alumnos (...) Resulta frecuente que uno o dos hacen el trabajo del resto (...) La forma que la maestría califica (nota grupal), hace que una porción de alumnos sobrevivan, curso tras curso, en base al trabajo de otros.

Hay una cultura de falso compañerismo y nadie quiere ser el malo, el problema es que algunos alumnos aprenden que siendo lentos en responder a correos, apareciendo ocupados, y contribuyendo lo mínimo, es más fácil, y no hay ninguna consecuencia, ya que otro termina haciendo el trabajo, para salvar la nota (...) (A6).

- En cuanto al desarrollo del sentido crítico, el análisis y resolución de problemas, aunque una mayoría se muestra a favor de estas capacidades, llama la atención un $43 \%$ de alumnos que relativizan la importancia de la capacidad de "observación y comprensión de problemas, para la generación de alternativas y soluciones idóneas", sobre todo porque son alumnos de una especialidad que trabaja con problemas sociales.

\section{Nivel de desarrollo de las capacidades de AE en los alumnos de la MGS-D}

Menos de la mitad de los alumnos consultados se autoperciben con capacidades para realizar un AE. Un $37 \%$ está en proceso y un $9 \%$ afirma no contar con estas capacidades.

Muestran mejor desarrollado en la "motivación para aprender" y el "análisis y resolución de problemas" dato que explica, en parte, la relativa importancia que le asignaron en la pregunta anterior.

Las capacidades menos logradas son "aprendizaje con otros y de otros", "autorregulación de su proceso de aprendizaje", la "gestión de información y conocimiento" y "conoce cómo aprender". 
Tabla 3. Autopercepción sobre capacidades que forman parte de su desempeño actual Fuente: Encuesta online a estudiantes de la MGS-D.

\begin{tabular}{|c|c|c|c|c|c|}
\hline Áreas de capacidad & Autoperc & epció & & & \\
\hline Está motivado a aprender & $8 \%$ & $39 \%$ & $49 \%$ & $0 \%$ & $4 \%$ \\
\hline Conoce cómo aprender & $8 \%$ & $48 \%$ & $38 \%$ & $0 \%$ & $6 \%$ \\
\hline Autorregula su aprendizaje & $8 \%$ & $51 \%$ & $37 \%$ & $0 \%$ & $4 \%$ \\
\hline Gestiona información y conocimiento & $10 \%$ & $49 \%$ & $37 \%$ & $1 \%$ & $3 \%$ \\
\hline Aprende con otros y de otros & $14 \%$ & $45 \%$ & $36 \%$ & $2 \%$ & $3 \%$ \\
\hline Desarrolla sentido crítico & $12 \%$ & $42 \%$ & $42 \%$ & $0 \%$ & $4 \%$ \\
\hline Analiza y resuelve problemas & $5 \%$ & $41 \%$ & $45 \%$ & $1 \%$ & $8 \%$ \\
\hline
\end{tabular}

No cuento con esa capacidad; $\square$ En proceso de lograr esa capacidad; $\square$ Sí cuento con esa capacidad; No requiero esa capacidad; $\square N R$.

Nótese un $14 \%$ de estudiantes que afirman carecer completamente de la capacidad "Aprender con otros" que es fundamental no solo para un AE, sino para su rol profesional.

Se encuentra una correlación positiva (del 20 al 25\%) en las respuestas de los alumnos que relativizan la importancia de las capacidades "autorregulación" y "aprendizaje con otros", y el menor o nulo avance en el logro de estas capacidades, lo cual advierte de alumnos que enfrentan problemas en competencias fundamentales y que no aspiran a su desarrollo al interior de la maestría (actitud poco favorable a un cambio).

Algunas de las debilidades encontradas en el desarrollo de los alumnos son confirmadas por los docentes:

Estos chicos no están acostumbrados a leer mucho, el nivel es pobre, copian pegan y hacen un pobre análisis (DC3).

Estamos ante un público que viene de una vida de implementación de proyectos y que la MGS les plantee investigación es un cambio de chip (DC4).

Hay un buen grupo de alumnos que no planifica y lo que hacen es mandarte siempre excusas, no se organizan para entregar (...) (DC6).

La mayoría no tiene esta capacidad, y cuando tú los desapruebas reclaman (DC2). 
El alumno no lo valora lamentablemente (...) En muy pocas experiencias veo que el alumno lo valora, pero tampoco tienen tiempo (...) (DC6).

No se ha encontrado correlación entre capacidad alcanzada y edad de los participantes, variable que Hussein-Farraj, Barak y Dori (2012) recomiendan explorar por el distinto relacionamiento que enfrenta la población más joven con las tecnologías de información y el estudio a distancia.

\section{Responsabilidades en el desarrollo de capacidades de AE}

Más de la mitad de los estudiantes consultados han manifestado autocríticamente su responsabilidad individual en el desarrollo de las capacidades de AE propuestas. En otros casos han evidenciado que es una responsabilidad compartida. La revisión teórica indica que lograr un $\mathrm{AE}$ es una responsabilidad compartida entre el programa de formación (enseñanza) y la actuación del alumno. Algunos alumnos son conscientes que por ser adultos y por haber atravesado la educación superior, no pueden demandar esta formación a la maestría. Inclusive dicen "tener vergüenza de comunicar sus debilidades".

En los docentes hay una percepción heterogénea. Hay un mayor acuerdo en que las capacidades "motivación", "aprendizaje con otros" y "desarrollo de un sentido crítico" son responsabilidades compartidas entre el alumno y la maestría. Pero, atribuyen mayor responsabilidad a los alumnos en el desarrollo de capacidades metacognitivas.

El alumno tiene que venir con esa capacidad (de aprender) (DC2).

Es responsabilidad del estudiante (capacidad de aprender) (...) Se debe poner así en los formularios (de ingreso) (DC14).

Tabla 4. ¿Quién(es) es (son) responsable(s) de lograr un AE en los alumnos de la maestría?

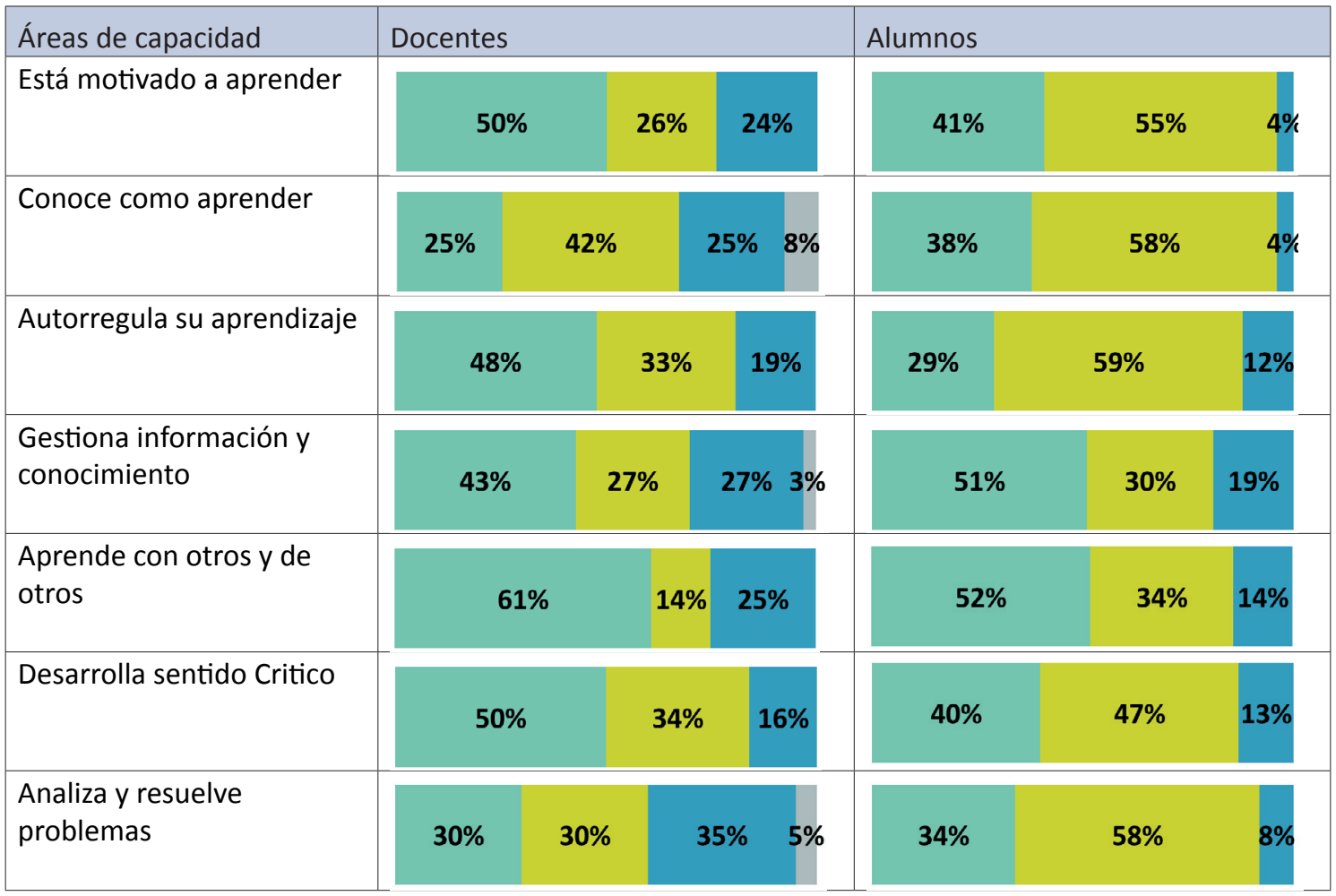

$\square$ Ambos son responsables; $\square$ Los estudiantes son responsables; $\square$ La maestría es responsable; $\square$ Ninguno. 
Estos docentes (42\%) no son muy conscientes de la importancia del proceso de enseñanza en este logro. En el otro extremo un $25 \%$ de docentes, algunos muy críticos del programa de maestría, señalan que la responsabilidad principal es de la MGS-D.

Como se observa una cuarta parte de docentes, aproximadamente, considera que el aprender estratégicamente es una responsabilidad individual de los alumnos.

\section{Discusión}

La validación de un perfil de capacidades para aprender estratégicamente, más allá de la buena voluntad de los actores consultados, implica debatir:

¿En qué medida es pertinente incluir competencias genéricas de aprendizaje estratégico en el nivel posgrado? Probablemente el sentido común señale que no corresponde, sin embargo, la investigación revela que esta necesidad existe, en el caso de estudio, y probablemente en escuelas con similares condiciones, que no son pocas en América Latina. El origen del problema sin duda está en la formación básica, pero también en el débil conocimiento que tienen las escuelas y docentes sobre la población de estudiantes admitidos en el posgrado, acerca de las capacidades transversales que traen para al nuevo proceso de aprendizaje, y sobre sus percepciones y avances en el proceso mismo de aprender. El problema será mayor al no incluir dichas competencias genéricas en la formación de posgrado, porque el alumno no podrá aprovechar con suficiencia las nuevas materias, y porque se trata del último eslabón de la cadena educativa en la formación profesional.

Entonces, ¿qué tipo de responsabilidad alcanza a los programas de maestría o posgrado ( $y$ al proceso de enseñanza) en la búsqueda de un $A E$ contextualizado en las necesidades actuales?

Los actores consultados parecen estar de acuerdo en que se trata de una responsabilidad compartida entre los programas de formación (que involucra a docentes) y los alumnos. La respuesta de coordinadores de la MGS-D y de diseñadores no es la misma: ellos revelan categóricamente que son competencias de responsabilidad individual de los alumnos.

Las condiciones y argumentos expuestos en este estudio indican la necesidad de asumir el reto, que no solo implica la revisión de los procesos de enseñanza, sino también de los aspectos de gestión como convocatoria, selección, inducción o bien tutoría o acompañamiento más individualizado.

Enseñar estratégicamente a distancia aparece en el estudio como un proceso complejo y poco conocido desde los propios docentes. Tres aspectos críticos parecen explicar esta complejidad: La carencia de entrenamiento docente, el débil acompañamiento al proceso formativo, y la ausencia de estrategias de aprendizaje.

Desde los alumnos se evidencia autocrítica, preocupación, disposición, temor y hasta vergüenza, sentimientos que bien pueden constituir el punto de partida de un abordaje más claro y directo de la responsabilidad del alumno en el AE. 


\section{Conclusiones}

Los resultados del estudio confirman que la abundante reflexión teórica, que propone y orienta la incorporación de algunas competencias genéricas para favorecer un aprendizaje continuo y autónomo ( $\mathrm{AE}$ ) en la educación superior, está en total sintonía con las necesidades prácticas sentidas por alumnos y docentes del programa de maestría consultado.

Se ha validado una propuesta de capacidades transversales donde la motivación, el conocimiento de cómo aprender, la autorregulación y el trabajo colaborativo, entre otras capacidades, han sido asumidas como relevantes en un programa de estudio de posgrado, no solo porque hay acuerdo de alumnos y docentes, que es fundamental, sino porque se ha reconocido un nivel parcial de logro de tales capacidades en más de la mitad de los alumnos consultados, y porque también se ha comprobado que alumnos y docentes se reconocen responsables.

Tres razones condicionan y refuerzan la idea de que el $A E$ no solo es válido sino necesario en su implementación, en la educación superior de posgrado, a distancia:

a. Las características educativas de los alumnos que observan déficits en su formación básica y de pregrado.

b. La identificación de debilidades en la relación educativa a distancia sobre el cual se profundizara en la segunda fase del estudio.

c. Necesidad de una conciencia clara en docentes, coordinadores, diseñadores y directivos sobre la corresponsabilidad para lograr el AE.

Es necesario revisar la noción y estrategia de implementación del $A E$, dimensionando los diferentes factores que intervienen, el rol clave del proceso de enseñanza, y las características y responsabilidades de los estudiantes.

Solo la acción consciente y deliberada de enseñar y aprender puede contribuir a avanzar en el reto de formar profesionales estratégicos para una sociedad en constante renovación.

\section{Referencias bibliográficas}

Aebli, H. (1991). Factores de la enseñanza que favorecen el aprendizaje autónomo. Madrid: Narcea.

Argüelles, D. y Nagles, N. (2010). Estrategias para promover procesos de aprendizaje autónomo. Bogotá: Universidad EAN \& Alfaomega.

Bacchetta, S. (2013). On Wedemeyer's Theory of Independent Study. Instructional Design Hub [Web]. Recuperado de http://instructionaldesignhub.blogspot. com/2013/01/on-wedemeyers-theory-of-independent_17.html [Último acceso: noviembre 2017].

Badia, A. y Monereo, C. (2008). La enseñanza y el aprendizaje de estrategias de aprendizaje en entornos virtuales. En Coll, C. \& Monereo, C, (2008). Psicología de la Educación Virtual. 
Cerezo, R., Núñez, J., Fernández, E., Suárez, N. y Tuero, E. (2011). Perspectiva Educacional, Formación de Profesores. Red de Revistas Científicas de América Latina \& el Caribe, España \& Portugal. Pontificia Universidad Católica de Valparaíso Chile. Recuperado de http://www.redalyc.org/articulo.oa?id=333327289002 [Último acceso: noviembre 2017].

Fernández, R. y Wompner, F. (2007). Aprender a aprender. Un método valioso para la educación superior. Observatorio de la Economía Latinoamericana. MPRA Munich Personal RePEc, 3613(1), 1-10. Recuperado de http://mpra.ub.uni-muenchen. de/3613/1/MPRA_paper_3613.pdf [Último acceso: noviembre 2017].

Gargallo, B. (2011). Un aprendiz estratégico para una nueva sociedad. XII Congreso de internacional de teoría de la educación. Universidad de Barcelona. Recuperado de http://www.cite2011.com/Comunicaciones/Escuela/066.pdf [Último acceso: noviembre 2017].

González, M. y Cabrera, I. (2013). Aprendizaje estratégico en la universidad: propuesta de asignatura para el currículo optativo/electivo. Revista de Pedagogía, 34(94), 261-281. Recuperado de http://www.redalyc.org/articulo.oa?id=65930105004 [Último acceso: noviembre 2017].

González, P. (2011). Curso Cátedra Unadista [material didáctico]. Bogotá: Universidad Nacional Abierta y a Distancia. Recuperado de http://datateca.unad.edu.co/ contenidos/434206/434206/index.html [Último acceso: noviembre 2017].

Huertas, M. (2007). Aprendizaje estratégico, una necesidad del siglo XXI. Revista Iberoamericana de educación. Recuperado de http://www.rieoei.org/1541.htm [Último acceso: noviembre 2017].

Hussein-Farraj, R., Barak, M. \& Dori, Y. J. (2012). Lifelong learning at the technion: Graduate students' perceptions of and experiences in distance learning. Interdisciplinary Journal of E-Learning and Learning Objects, 8. https://doi.org/10.28945/1731

MacDougall, M. (2010). Diez consejos para promover el aprendizaje autónomo y el compromiso efectivo al enseñar contenidos complejos. Revista de Educación en Ciencias y Salud, 7(1). Recuperado de http://www2.udec.cl/ofem/recs/ anteriores/vol712010/artrev71h.pdf [Último acceso: noviembre 2017].

Martín, E. (2008). Aprender a aprender: clave para el aprendizaje a lo largo de la vida. Revista Participación educativa. Recuperado de http://www.cece.gva.es/consell/ docs/jornadas/conferenciaelenamarti.pdf

Martín, E. (2010). Aprender a aprender: Una competencia básica entre las básicas. Madrid: Universidad Autónoma de Madrid. Recuperado de http://www.cece.gva. es/consell/docs/jornadas/conferenciaelenamarti.pdf [último acceso: noviembre 2017].

Martín Cuadrado, A. (2011). Competencias del estudiante autorregulado y los estilos de aprendizaje. Revista Estilos de Aprendizaje, 8(4), 136-148. Recuperado de http://learningstyles.uvu.edu/index.php/jls/article/view/68/44 [Último acceso: noviembre 2017]. 
Tsai, M.-J. (2009). The Model of Strategic e-Learning: Understanding and Evaluating Student e-Learning from Metacognitive. Perspectives. Educational Technology \& Society, 12(1), 34-48. Recuperado de http://www.ifets.info/journals/12_1/4.pdf [Último acceso: noviembre 2017].

Monereo, C. (coord.), Castelló, M., Clariana, M., Palma, M. y Pérez, M. (1999). Estrategias de enseñanza y aprendizaje. Formación del profesorado y aplicación en la escuela. Barcelona: Graó.

Monereo, C. y Badia, A. (2013). Aprendizaje estratégico y tecnologías de la información y la comunicación: Una revisión crítica. Teoría de la Educación. Educación y Cultura en la Sociedad de la Información, 14(2), 15-41. Recuperado de http://www. redalyc.org/pdf/2010/201028055002.pdf [Último acceso: noviembre 2017].

Pozo, J., Monereo, C. y Castelló, M. (2001). El uso Estratégico del Conocimiento. Recuperado de http://www.academia.edu/3264771/EL_USO_ESTRAT\%C3\%89GICO_DEL_ CONOCIMIENTO1 [Último acceso: noviembre 2017].

Pozuelo, J. (2014). ¿Y si enseñamos de otra manera? Competencias digitales para el cambio metodológico. Caracciolos. Revista digital de investigación en docencia, 2(1). Recuperado de http://www3.uah.es/caracciolos/index.php/caracciolos/ article/view/17/36 [Último acceso: noviembre 2017].

Santos, R. y Cámara, M. (2010). Autonomy in distance learning: Reflections over the learner's role. $16^{\circ}$ Congresso Internacional de Educaçao a Distancia ABED. Recuperado de http://www.abed.org.br/congresso2010/cd/ing/252010174412. pdf [Último acceso: noviembre 2017].

Stake, R. E. (1998). Investigación con Estudio de Casos. 2da Edición. Madrid: Ediciones Morata.

Stojanovic, L. (1994). Bases teóricas de la educación a distancia. Informe de Investigaciones Educativas. Universidad Nacional Abierta, $3(1$ y 2), 11-45. Recuperado de http:// cursoampliacion.una.edu.ve/fundamentos/paginas/lily1994.pdf [Último acceso: noviembre 2017].

Sun, T. (2009). El Arte de la Guerra. Barcelona: Obelisco

Torrano, F. y Gonzales, M. (2004). El aprendizaje autorregulado: presente y futuro de la investigación. Revista Electrónica de Investigación Psicoeducativa. Recuperado de http://www.webdocente.altascapacidades.es/Aprendizaje\%20Autorregulado/ Art_3_27.pdf [Último acceso: noviembre 2017].

Unesco (1998). La educación superior en el siglo XXI: Visión y acción. Conferencia Mundial sobre la Educación Superior, París. Recuperado de http://www.unesco. org/education/educprog/wche/declaration_spa.htm [Último acceso: noviembre 2017].

Zimmerman, B. (2002). Becoming a Self-Regulated Learner: An Overview. Journal Taylor \& Francis Group content, 41(2), 64-70. https://doi.org/10.1207/ s15430421tip4102_2

Zulma, M. (2006). Aprendizaje autorregulado: el lugar de la cognición, la metacognición y la motivación. Estudios pedagógicos (Valdivia), 32(2), 121-132. https://dx.doi. org/10.4067/S0718-07052006000200007 
Artículo concluido el 25 de agosto de 2016

Salcedo Lobaton, E. (2017). Aprender estratégicamente: Percepciones de docentes y alumnos de un programa de maestría a distancia. REDU. Revista de Docencia Universitaria, 15(2), 143-162.

https://doi.org/10.4995/redu.2017.6541

\section{Elizabeth Salcedo Lobatón \\ Pontificia Universidad Católica del Perú \\ Maestría en Gerencia Social elysalce@gmail.com}

Profesora de la Maestría en Gerencia Social y de la Maestría en Educación de la Pontificia Universidad Católica del Perú. Doctoranda en Pedagogía UNED 